\title{
EFFECTIVENESS OF ELECTROTHERAPY IN CHRONIC PAINFUL DIABETIC NEUROPATHY
}

\author{
Alagianambi Shanmugam¹, Thayanithi Jayapackiam², Stephen Charles Bronson ${ }^{3}$, Loganathan Nandhini $^{4}$, Duvuru Shantharam ${ }^{5}$
}

${ }^{1}$ Associate Professor, Institute of Diabetology, Stanley Medical College, Chennai.

${ }^{2}$ Assistant Professor, Institute of Diabetology, Stanley Medical College, Chennai.

${ }^{3}$ Senior Resident, Institute of Diabetology, Stanley Medical College, Chennai.

${ }^{4}$ Postgraduate Student, Bharathidasan University, Trichy.

5Director and Professor, Institute of Diabetology, Stanley Medical College, Chennai.

\begin{abstract}
BACKGROUND

Diabetic neuropathy is the commonest complication of diabetes; more than $50 \%$ of people with type 2 diabetes of 10 years duration have been affected with diabetic neuropathy.(1) Chronic Distal Symmetrical Polyneuropathy (DSPN) and autonomic neuropathy are the common neuropathies and most studied neuropathies. $(2,3)$ DSPN is the most common clinical neuropathy of the diabetes population and up to $50 \%$ of DSPN patients may experience symptoms, most frequently burning pain, electrical or stabbing sensations, paresthesiae, hyperasthesiae and deep aching pain. Neuropathic pain is typically worse at night and the symptoms are most commonly experienced in the feet and lower limbs, although in some cases the hands may also be affected. Most of the painful DSPN patients may experience excruciating pain in early stages of neuropathy. Glycaemic control, preferably with insulin is the mainstay of prevention and delay the progression of diabetic neuropathies. Apart from glycaemic control, pregabalin and duloxetine have received regulatory approval for the treatment of neuropathic pain in diabetes and various nonpharmacological therapies had been tried to reduce the diabetic neuropathic pain. This study is to evaluate the effectiveness of electrotherapy in reducing the severity of pain and to assess the changes in vibration perception in diabetic neuropathy patients.

The objective of this patient is to evaluate the efficacy of electrical nerve stimulation for the management of chronic painful neuropathy in patients with type 2 diabetes.
\end{abstract}

\section{MATERIALS AND METHODS}

Sixty (60) painful diabetic neuropathy patients attending Diabetology Clinic at Government Stanley Medical College Hospital are submitted for 4 weeks of electrical stimulation using Neurostim (Portable electrical neuromuscular stimulation instrument). Effectiveness of pain reduction of electrical neurostimulation assessed in terms of pain scale and vibration perception before and after 4 weeks of Neurostim treatment.

\section{RESULTS}

The symptomatic response rate was fairly satisfactory. Almost $85 \%$ of painful neuropathic patients felt the symptomatic reduction in pain. Pain score reduced from $6.5 \pm 1.2$ to $3.8 \pm 1.0(\mathrm{P}<0.001)$ and improvement in vibration perception from $25.5 \pm 3.4$ to 21.6 $\pm 3.7(\mathrm{P}<0.001)$ and $25.5 \pm 3.2$ to $21.6 \pm 3.5(\mathrm{P}<0.001)$ on the right and left sides respectively. Vibration perception improved almost $20 \%$ after the Neurostim electrical stimulation over a period of 4 weeks. No local or systemic side effects were noticed with electrotherapy.

\section{CONCLUSION}

Our clinical observations suggest that electrotherapy is effective in reducing the pain associated with peripheral neuropathy. Electrotherapy showed modest improvement in the vibration perception also. This form of therapy may be a useful adjunctive modality when it is combined with a pharmacological agent to augment symptomatic relief and to avoid long-term diabetic neuropathic complications.

\section{KEYWORDS}

Diabetes Mellitus (DM), Distal Symmetrical Polyneuropathy (DSPN).

HOW TO CITE THIS ARTICLE: Shanmugam A, Jayapackiam T, Bronson SC, et al. Effectiveness of electrotherapy in chronic painful diabetic neuropathy. J. Evolution Med. Dent. Sci. 2017;6(17):1317-1322, DOI: 10.14260/Jemds/2017/286

Financial or Other, Competing Interest: None.

Submission 05-02-2017, Peer Review 17-02-2017,

Acceptance 20-02-2017, Published 27-02-2017.

Corresponding Author:

Dr. Alagianambi Shanmugam,

Associate Professor,

Institute of Diabetology,

Government Stanley Medical College,

Chennai-01.

E-mail:drshanmugaa@gmail.com

DOI: $10.14260 /$ jemds $/ 2017 / 286$

(c) $(\mathrm{i})($

\section{BACKGROUND}

Peripheral neuropathy is a common complication of diabetes, affecting more than $50 \%$ of patients with more than 10 years of diabetes duration. It has been estimated that up to $20 \%$ of patients with Diabetes suffer from painful neuropathy.(1,2) The pathogenesis of this pain is complex; treatment for this often debilitating condition is equally so. Unfortunately, painful diabetic neuropathy still remains a challenge to the treating physician. Numerous pain management protocols have been advocated to ameliorate neuropathic pain. $(3,4)$ These have included antidepressants, anticonvulsants, $\alpha$ adrenergic agonists, aldose reductase inhibitors, intravenous 
insulin, intravenous anaesthetics and topical counterirritants among others.(5) Even though various drugs are available to treat painful diabetic neuropathies, not all the patients are comfortable with available treatment options. These molecules may have their own adverse reactions and they would not correct the underlying aetiopathogenesis of diabetic neuropathies. Various non-pharmacological modalities such as electrotherapy(6) and acupuncture(7) had been tried to reduce the symptoms of painful diabetic neuropathy. Neurostimulation is a therapeutic activation of part of the nervous system using special electrodes. Electrodes are one of the key components of the neurostimulation, which deliver the electronic potentials to stimulate neurons. Neurostimulation is a chronic pain treatment option for people who suffer from various types of neuropathic pain.

There are a number of descriptive articles indicating that electrical stimulation may be an effective pain relieving modality.(8-10) Prompted by the beneficial effects of electrotherapy and mechanism by which electrostimulation relieves pain is distinct from currently available pharmacological therapies, we tried to evaluate the efficacy of such a therapy in painful diabetic neuropathy in our tertiary care hospital.

The digital biothesiometer- Vibrometer is an electronic instrument designed to measure the threshold of appreciation of vibration in human subjects. People who cannot sense pressure from monofilament have lost protective sensation and are at high risk for developing foot ulcers and future amputations. Vibrometer help us to quantify the vibration perception status of the individual and to classify them into risk group.

\section{MATERIALS AND METHODS}

\section{Study Patients}

Known diabetes patients attending Diabetology Clinic of Government Stanley Medical College Hospital with painful diabetic neuropathy participated in a study protocol approved by the Institutional Ethical Committee. Inclusion criteria for the study is type 2 diabetes patients with DPN and Age: 30 - 70 years. Exclusion criteria for the study is type 2 DM patients who are pregnant, lactating mother, chronic alcohol consumption and substance abuse, Hansen's Disease, anti-tuberculosis therapy, renal failure and patients with vascular insufficiency. Patients on steroids and chemotherapeutic agents were also excluded. Participants of some other studies were also excluded.

\section{Study Design}

As per the protocol, sixty (60) type 2 diabetes patients with painful diabetic neuropathy were selected for the study. On the initial visit, a detailed history and physical examination was performed and recorded as per the need.

A Stanford pain distress scale(11) was used to measure individual patient's pain intensity and limitations in Activities of Daily Living Scale (ADLs). Pain scales are based on selfreport and/or observational (behavioural) data. Self-report is considered primary and should be obtained if possible. Figure 1 shows pain scale image. Table 1 shows rating and pain level for painful diabetic neuropathy patients.

\begin{tabular}{|c|c|}
\hline Rating & Pain Level \\
\hline 0 & No Pain \\
\hline $1-3$ & $\begin{array}{r}\text { Mild Pain (Nagging, annoying, interfering little } \\
\text { with ADLs) }\end{array}$ \\
\hline $4-6$ & Moderate Pain (Interferes significantly with ADLs) \\
\hline $7-10$ & Severe Pain (Disabling; unable to perform ADLs) \\
\hline \multicolumn{2}{|c|}{ Table 1. Pain Score Scale and Rating } \\
\hline
\end{tabular}

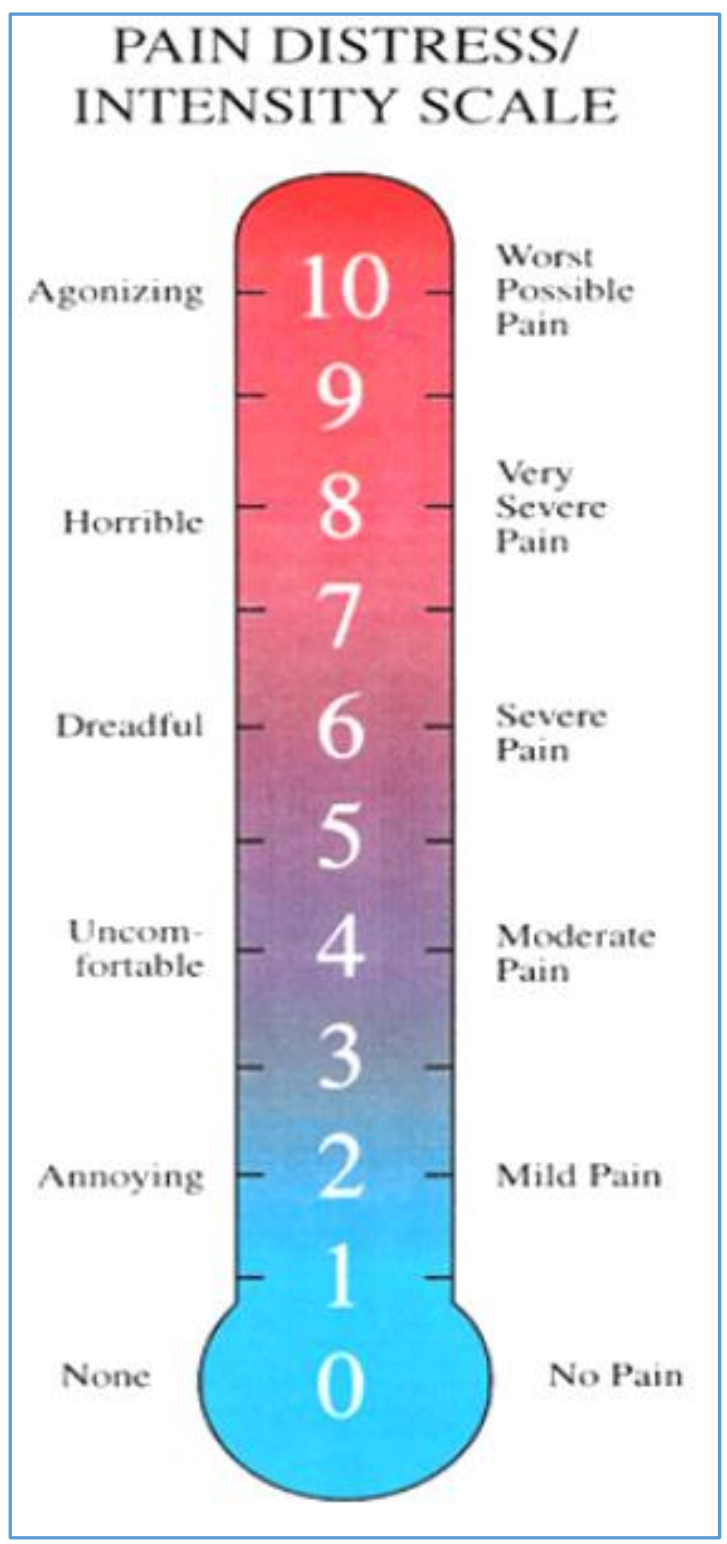

Figure 1. Pain Scale Image

Table 1. Rating and pain level for Painful Diabetic Neuropathy Patients.

A digital biothesiometer - vibrometer is the essential tool for larger fiber sensory nerve evaluation quantifies vibratory perception thresholds for evaluation of the Peripheral Neuropathy (PN). It has computer connectivity through USB cable and with the software supplied the vibrometer data are transferred to computer for storage and patient report generation. It is essential an "electronic tuning fork" whose vibration strength may be gradually increased until the threshold of vibration. The vibrometer is not far superior to a tuning fork in accuracy, but will detect neurological changes that are not disclosed with a tuning fork. 


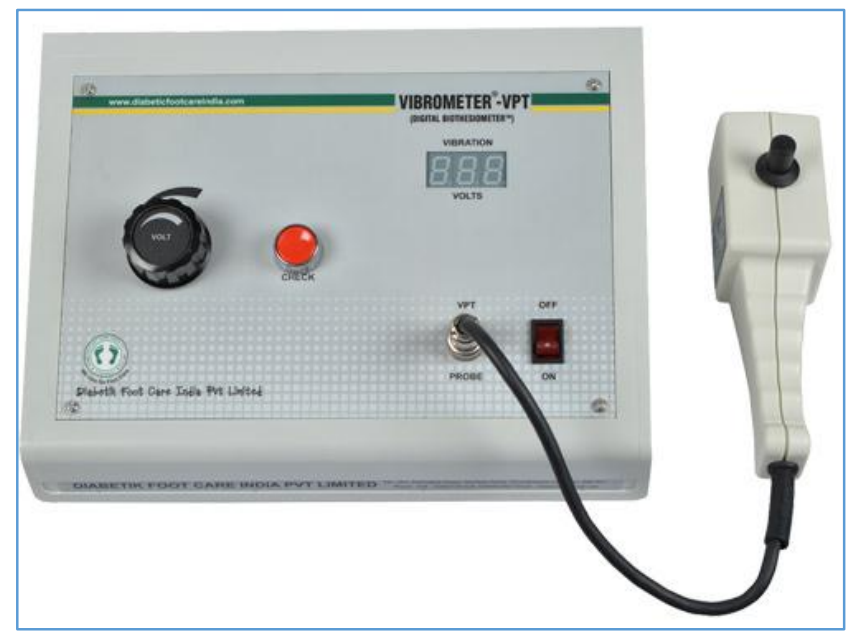

Figure 2. Vibrometer

Vibration perception threshold measured in six designated points of the foot and the mean value taken as the final vibration threshold value for calculations.

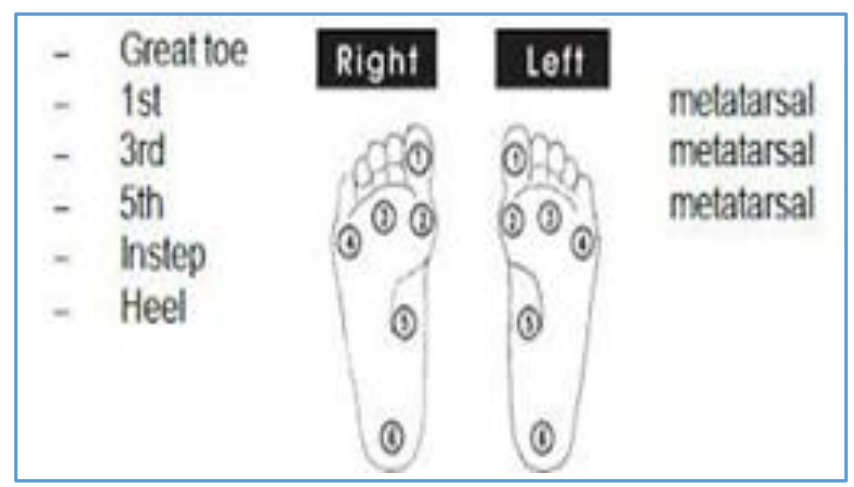

Figure 3. Biothesiometry Study

All study recruited patients have been subjected with biothesiometer vibration assessment and the subjective pain score before and after the electrotherapy.

Neurostim is a portable and rechargeable electronic neuromuscular stimulator instrument (Diabetik Foot Care India Pvt. Ltd.), which delivers mild electrical signals that produces tingling sensation on application. Neurostim delivers adjustable output voltage of 90 volts against a load of $4.7 \mathrm{~K}$. Ohms through a pair of two-inch conductive rubber electrodes, which are placed over the sole of the feet.

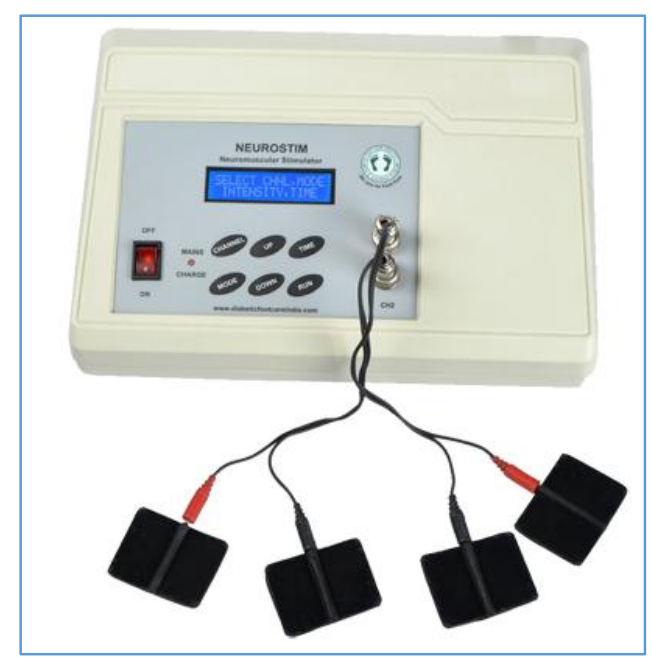

Figure 4. Neurostim Instrument
Each patient was individually instructed about the nerve stimulation by the investigator. Individual patient Neurostim electrical stimulation voltage fixed according to the patient's body impedance and perception up to the maximum of 90 voltages. If patient feels the comfortable nerve stimulation with the 25 voltage, patient's treatment is fixed with 25 voltages. On regular application of electrical neurostimulation, if patient felt discomfort like burning pain because of improvement, voltage reduced with lower level of patient perception and comfort. This assessment was done on every sitting before starting and fixing voltage on that day. The nerve stimulation was done for a period of 30 minutes for each patient for daily 5 days in a week for 4 weeks (20 sittings).

After four weeks of Neurostim electrical stimulation, all the study group individuals subjected for biothesiometer score and subjective pain score.

\section{Statistics}

The data was analysed and presented as mean, standard deviation, median and range and for categorical data (sex and pain level) as frequency and percentage. Wilcoxon Signed Rank test was done to compare the pre-treatment and posttreatment measurements of Biothesiometer, Neurostimulation and pain distress scale. The score on pain distress was grouped as given in Table 1 and tested for Statistical Significance using Marginal Homogeneity test. All analysis was done in SPSS version 17. A P value of less than 0.05 was considered as statistically significant.

\section{RESULTS}

The data collected from the 60 Type 2 DM patients with painful (peripheral) diabetic neuropathy were analysed and the results are presented here.

Demographic Characteristics - Age of these patients ranged from 35 to 68 years with a mean (SD) of 55.1 (7.2) years. There were $47(78.3 \%)$ females and the duration of diabetes ranged from 1 to 18 years with a mean (SD) of 8.7 (4.4) years. These findings are given in -

\begin{tabular}{|c|c|}
\hline Characteristics & Statistics \\
\hline Age (years) & $55.1 \pm 7.2($ Mean \pm SD) \\
\hline Sex No. (\%) & $\begin{array}{c}\text { Males: } 13 \text { (21.7) Females: } 47 \\
(78.3)\end{array}$ \\
\hline $\begin{array}{c}\text { Duration of diabetes } \\
\text { (years) }\end{array}$ & $8.7 \pm 4.4($ Mean \pm SD $)$ \\
\hline $\begin{array}{r}\text { Table } 2 . \\
\text { of Demog }\end{array}$ & $\begin{array}{l}\text { iptive Statistics } \\
\text { c Characteristics }\end{array}$ \\
\hline
\end{tabular}

Biothesiometer Reading (Vibration Perception Threshold- VPT) - VPT for right and left legs ranged from 20 to 37 volts during pre-treatment assessment, whereas at the time of post-treatment assessment (4 weeks after the treatment) it ranged from 17 to 35 volts. In the present study, VPT more than $15 \mathrm{~V}$ is taken as having presence of neuropathy. Therefore, all patients before and after treatment had neuropathy, but there were significant reduction in the values at the post-treatment assessment as compared to pre-treatment. These results are given in Table 3 and Figure 5. 


\begin{tabular}{|c|c|c|c|}
\hline \multirow{2}{*}{ Leg } & Pre-Treatment & Post-Treatment & Comparison of Pre- and Post-Treatment \\
& Mean \pm SD Median Min - Max & Mean \pm SD Median Min - Max & $<0.001$ \\
\hline \multirow{2}{*}{ Right } & $25.5 \pm 3.425 .0$ & $21.6 \pm 3.720 .517-35$ & $<0.001$ \\
\hline Left & $20-37$ & $21.6 \pm 3.521 .017-34$ & $<$ \\
\hline \multicolumn{2}{|r|}{ Table 3. Biothesiometer Reading (VPT) during Pre- and Post-Treatment Evaluation } \\
\hline
\end{tabular}

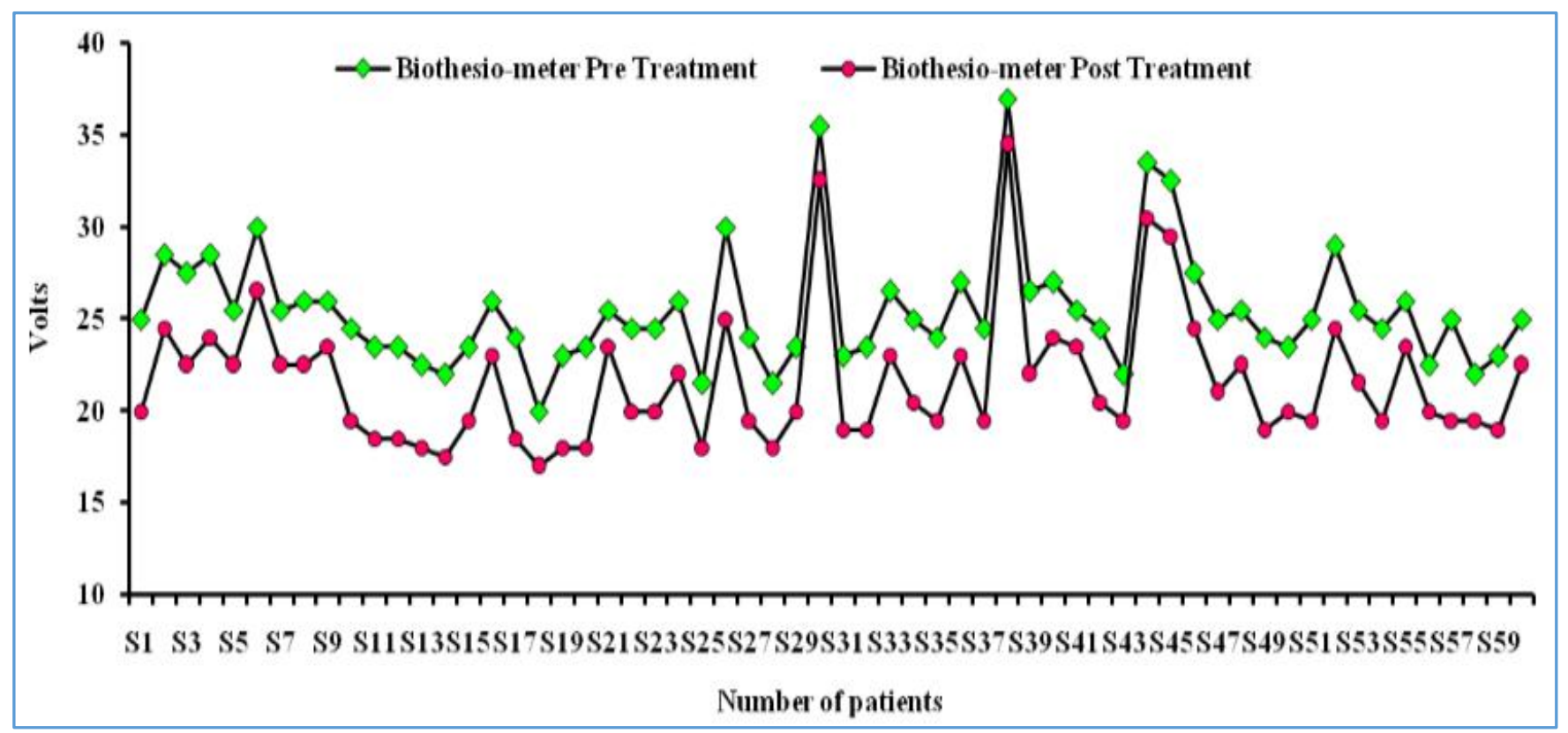

Figure 5. Assessment of Vibration Perception Threshold before and after Treatment

Effect of Neurostimulation Treatment in Painful Diabetic Neuropathy - Assessment was done at baseline and four weeks after the treatment to evaluate the effect of nerve stimulation in reducing the severity of pain in these patients. There was significant reduction in pain at the post-treatment evaluation as compared to pre-treatment.

\begin{tabular}{|c|c|c|}
\hline Pre-Treatment & Post-Treatment & $\begin{array}{c}\text { Comparison of } \\
\text { Pre- and Post- } \\
\text { Treatment } \\
\text { P value }\end{array}$ \\
\hline $\begin{array}{c}\text { Mean } \pm \text { SD Median } \\
\text { Min - Max }\end{array}$ & $\begin{array}{c}\text { Mean } \pm \text { SD } \\
\text { Median Min - Max }\end{array}$ & $<0.001$ \\
\hline $\begin{array}{c}41.8 \pm 7.340 .0 \\
32-67\end{array}$ & $\begin{array}{c}35.4 \pm 6.934 .0 \\
27-63\end{array}$ & $<3$ (In Voltage) \\
\hline \multicolumn{2}{|c|}{ Table 4. Effect of Neurostimulation Treatment (n) }
\end{tabular}

Pain Distress Scale - The median pain at the pre-treatment level was 6 , which was reduced significantly $(p<0.001)$ to 4 at the post-treatment level as given in Table $5 \mathrm{a}$. At the pre- treatment assessment, the score ranged from 4 to 8 (Table $5 a)$, which means that there was no patient in the no pain and mild pain categories; $65 \%$ had moderate pain and the remaining had severe pain. At the post-treatment evaluation, there was no patient in the no pain and severe pain categories, $81.7 \%$ in the moderate category and the remaining in the mild category of pain. The reduction in the pain score at the post-treatment evaluation was statistically significant $(\mathrm{p}<0.001)$. These results are given in Table $5 \mathrm{~b}$.

\begin{tabular}{|c|c|c|}
\hline Pre-Treatment & Post-Treatment & Comparison of \\
\hline $\begin{array}{c}\text { Mean } \pm \text { SD: } 6.5 \pm 1.2 \\
\text { Median: } 6.0 \text { Min - } \\
\text { Max: } 4-8\end{array}$ & $\begin{array}{c}\text { Mean } \pm \text { SD: } 3.8 \pm 1.0 \\
\text { Median: } 4.0 \text { Min.- } \\
\text { Max: } 2 \text { - } 6\end{array}$ & $\begin{array}{c}\text { Pre- and Post- } \\
\text { Treatment } \\
\text { P value: }<0.001\end{array}$ \\
\hline
\end{tabular}




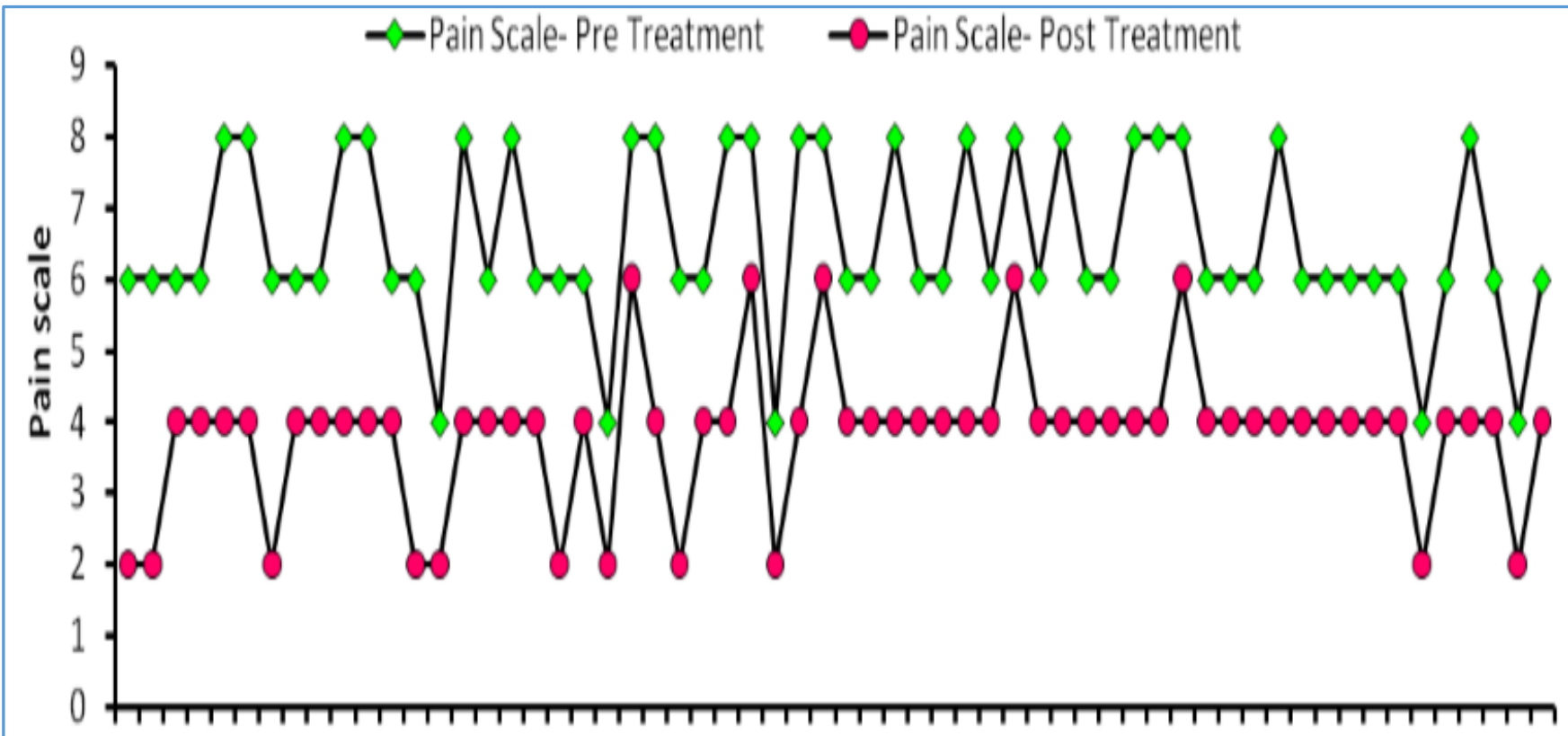

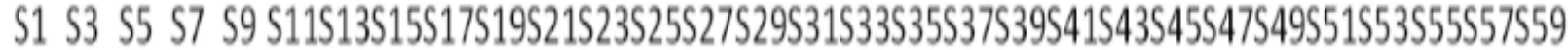

\section{Number of patients}

Figure 6. Assessment on each Painful Diabetic Neuropathy Patient's Pain Score before and after Treatment

\begin{tabular}{|c|c|c|}
\hline $\begin{array}{c}\text { Rate of Pain } \\
\text { (Value) }\end{array}$ & $\begin{array}{c}\text { Pre-Treatment } \\
\text { No. (\%) }\end{array}$ & $\begin{array}{c}\text { Post-Treatment } \\
\text { No. (\%) }\end{array}$ \\
\hline No pain (0) & 00 & 0 \\
\hline Mild pain (1 - 3) & 00 & $11(18.3)$ \\
\hline Moderate pain (4 - 6) & $39(65.0)$ & $49(81.7)$ \\
\hline Severe pain (7 - 10) & $21(35.0)$ & 00 \\
\hline Table 5b. Distribution of Pain Levels of Study Subjects \\
during Pre- and Post-Treatment Evaluation \\
\hline \multicolumn{2}{|c}{}
\end{tabular}

No local or systemic side effects were noticed with electrotherapy. There was no discernible improvement or deterioration of neurological signs during the study period.

\section{Metabolic Control}

Patients were free of symptoms of uncontrolled diabetes and had stable body weight, but their glycosylated haemoglobin levels were in the "poor" range (HbA1c more than 7\%). No attempt was made to modify medical treatment during the study period.

\section{CONCLUSION}

The symptomatic response rate was fairly satisfactory. Almost $85 \%$ of the patients' experiences benefits in the pain symptoms. Vibration perception improved almost $20 \%$ after the Neurostim electrical stimulation over a period of 4 weeks. By the improvement in perception, there was a significant reduction in Neurostim stimulation voltage during the course of the treatment. After 4 weeks, more than $10 \%$ drop in stimulation voltage required for the patient's perception. There is significant reduction in pain; almost $40 \%$ reduction in pain scale seen in our study group. A more impressive observation was that no patient felt severe pain in pain score scale after the Neurostim electrical stimulation.

\section{DISCUSSION}

When compared with pre- and post-treatment, significant reduction in pain scale and modest improvement in vibration perception occurred with electrotherapy. By reducing the pain and improving the vibration perception, the future risk of ulceration has been reduced in the high risk foot by electrotherapy. It may be postulated that patients receiving a stimulation device for treatment of subjective pain may have been exposed to a powerful placebo effect. This has been reported in other studies also. In their study of phantom limb pain, Finsen et al, found that while there was a considerable placebo effect the functioning stimulation units provided significantly more pain relief.

What is the mechanism of symptomatic relief with electrotherapy? Our clinical study does not provide any direct clue. However, considering that microangiopathic changes, reduced blood flow, nerve oxygen tension and other vascular factors all contribute to the pathogenesis(12) of diabetic neuropathy, it is conceivable that our treatment affected the circulatory status and improved the oxygen tension in peripheral nerves. Studies of the effects of transcutaneous electrotherapy on wound healing also suggest that such treatment could improve tissue circulation,(13) although the equipment and treatment parameters used were different from those used in our study. In addition some of the electrotherapy-induced neurophysiological changes(14,15) such as nerve conduction latency, mechanical pain threshold, nociceptive flexion reflex threshold and somatosensory evoked potentials could have contributed to the observed benefits.

One of the potential shortcomings of this study was the lack of a control group. In summary, our clinical observations suggest that electrotherapy may be a useful non-invasive, non-pharmacological treatment modality for the 
management of painful peripheral neuropathy in patients with type 2 diabetes. This form of therapy may be combined with a pharmacological agent to augment symptomatic relief. If confirmed in a larger series of cases and possibly in a multicentre study, these findings may offer new perspectives for the treatment of diabetic neuropathy.

\section{REFERENCES}

[1] Pop-Busui R, Lu J, Brooks MM, et al. Impact of glycemic control strategies on the progression of diabetic peripheral neuropathy in the bypass angioplasty revascularization investigation 2 diabetes (BARI 2D) cohort. Diabetes Care 2013;36(10):3208-15.

[2] Callaghan BC, Kerber KA, Lisabeth LL, et al. Role of neurologists and diagnostic tests on the management of distal symmetric polyneuropathy. JAMA Neurol 2014;71(9):1143-9.

[3] Dyck PJ, Albers JW, Andersen H, et al. Diabetic polyneuropathies: update on research definition, diagnostic criteria and estimation of severity. Diabetes Metab Res Rev 2011;27(7):620-8.

[4] Oyibo SO, Prasad YD, Jackson NJ, et al. The relationship between blood glucose excursions and painful diabetic peripheral neuropathy: a pilot study. Diabet Med 2002;19(10):870-3.

[5] Pop-Busui R, Boulton JMA, Feldman EL, et al. Diabetic neuropathy: a position statement by the American diabetes association. Diabetes Care 2017;40(1):13654.

[6] Armstrong DG, Lavery LA, Fleischli JG, et al. Is electrical stimulation effective in reducing neuropathic pain in patients with diabetes? J Foot Ankle Surg 1997;36(4):260-3.
[7] Ewins DL, Vileikyte L, Borg-Costanzi J, et al. A novel treatment for painful neuropathy. In: diabetic, new concepts and insights. Hotta N, Greene DA, Ward JD, (eds). Excerpta medica 1995:405-8.

[8] Finsen V, Persen L, Lovlien, M, et al. Transcutaneous electrical nerve stimulation after major amputation. J Bone Joint Surg 1988;70(1):109-12.

[9] Lewis D, Lewis B, Sturrock RD. Trascutaneous electrical nerve stimulation in osteoarthrosis: a therapeutic alternative. Rheum Dis 1984;43(1):47-9.

[10] Stabile ML, Mallory M. The management of postoperative pain in total joint replacement: transcutaneous electrical nerve stimulation is evaluated in total hip and knee patients. Orthop Rev 1978;7:121-3.

[11] Pain scalehttps://lane.stanford.edu/portals/cvicu/HCP_Neuro_ Tab_4/0-10_Pain_Scale.pdf

[12] Stevens MJ, Feldman EL, Greene DA. The aetiology of diabetic neuropathy: the combined roles of metabolic and vascular defects. Diabet Med 1995;12(7):566-79.

[13] Baker LL, DeMuth SK, Chambers R, et al. Effects of electrical stimulation on wound healing in patients with diabetes ulcers. Diabetes Care 1997;20(3):40512.

[14] Walsh DM, Foster NE, Baxter GD, et al. Transcutaneous electrical stimulation relevance of stimulation parameters to neurophysiological and hypoalgesic effects. Am J Phys Med Rehabil 1995;74(3):199-206.

[15] Janko M, Trontelj JV. Transcutaneous electric nerve stimulation: a microneurographic and perceptual study. Pain 1980;9(2):219-30. 